\title{
Analysis of jitter influence in fast frequency measurements
}

\author{
Oleg Sergiyenko ${ }^{\mathrm{a}, *}$, Daniel Hernández Balbuena ${ }^{\mathrm{b}}$, Vera Tyrsa ${ }^{\mathrm{c}}$, Patricia Luz A. Rosas Méndez ${ }^{\mathrm{b}}$, \\ Moises Rivas Lopez ${ }^{\mathrm{a}}$, Wilmar Hernandez ${ }^{\mathrm{d}}$, Mikhail Podrygalo ${ }^{\mathrm{e}}$, Alexander Gurko ${ }^{\mathrm{e}}$ \\ ${ }^{a}$ Engineering Institute of Autonomous University of Baja California, Blvd. Benito Juárez y Calle de la Normal S/N, Col. Insurgentes Este, 21280, Mexicali, BC, Mexico \\ ${ }^{\mathrm{b}}$ Engineering Faculty of Autonomous University of Baja California, Blvd. Benito Juárez y Calle de la Normal S/N, Col. Insurgentes Este, 21280, Mexicali, BC, Mexico \\ ${ }^{c}$ Polytechnic University of Baja California, Mexico \\ d Polytechnic University of Madrid, Spain \\ 'Kharkov National Highway and Automobile University, Ukraine
}

\section{Keywords:}

Frequency measurement

Noise sources

Timing jitter

Uncertainty

Measured value approximation by mediant

fractions

\begin{abstract}
A B S T R A C T
This paper presents a theoretical analysis of possible jitter impact in application of numeric criterion for fast measurement of frequency by coincidence principle. The primary goal is the generation of a signal containing a known amount of each jitter components. This signal was used for testing signals with regular pulse trains. Initially, jitter components are analyzed and modeled individually. Next, sequences for combining different kinds of jitter are modeled, simulated and evaluated. Jitter model simulation in Matlab is utilized to show the independence of frequency measurement results on the total jitter present in the reference and desired pulse trains independently. A good agreement between previously introduced theory of fast measurement of frequency and simulation in jitter presence is verified; these results allows to engineers use the numeric criterion for fast measurement of frequency in spite to interactions among jitter components in various applications for frequency domain sensors.
\end{abstract}

\section{Introduction}

It is well known that many practical applications in various fields of modern electronic technology, mentioned in $[1$, p. 136], are strongly dependant on frequency measurement exactitude. Also it is well known [2] that the best way for correct frequency estimation is the long term signal observation with Allan deviation curve registering. The necessity of Allan's curve it is caused, first of all, by the presence in any electrical pulse train of such natural phenomena like a jitter. The rigorous definition and systematic analysis of the jitter phenomena will be given below, in Section 3. Now we just will consider the jitter like arbitrary displacement of the electrical pulse from its theoretically considered position. This displacement, as shown in [3, see Fig. 2 on p. 2], affects both pulse parameters: its amplitude and position in time domain. Due to frequency

\footnotetext{
* Corresponding author. Tel.: +526865664150.

E-mail address: srgnk@iing.mxl.uabc.mx (0. Sergiyenko).
}

measurement method it is also important to note that both of pulse trains, unknown and reference, has jitter. The mentioned circumstances lead to that the coincidences between two pulse trains, unknown and reference (see Fig. 1), might be difficult to register by recently known electronic devices. But in [1] such coincidence existence is necessary condition for theoretical method functioning. In a worst case, the simple coincidence between two overlapped in time domain pulses can be missed because of jitter and own time delay in input-output of the \&-gate. So, it is evident the importance of the research and analysis of jitter influence in fast frequency measurement method offered in [1]. Firstly, will remind some basic points of this theoretical method.

\section{Fast frequency measurement method: brief description}

In [1] it is presented detailed description of the method for fast frequency measurement based on the direct comparison of two regular independent trains of narrow 


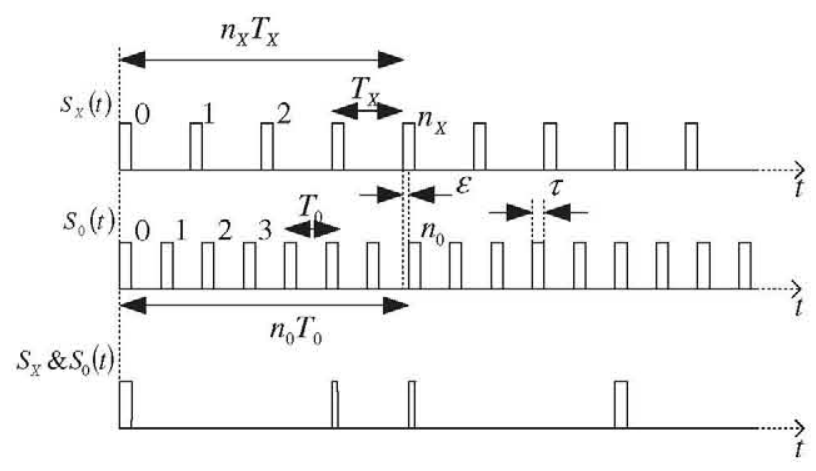

Fig. 1. Principle of frequency measurement for pulse coincidence.

pulses. In this method the stop event for the frequency measurement process is a numeric condition on number of pulses counted in the train of unknown frequency. Stop measurement condition is not an electronic detected event like in other methods is used [4-6].

In this method, an unknown frequency is measured by comparing it with a standard frequency. The zero crossings of both sinusoidal signals are detected, and a pulse is generated at each negative to positive crossing. Two regular independent trains of narrow pulses with different frequency are generated.

The trains of narrow pulses with unknown and standard frequency are compared for coincidence using an \&-gate. A coincidence pulse train is generated (Fig. 1), and an appropriate pulse in this train can be used as trigger to start a pair of digital counters (start event).

Each original pulse train is applied to different counters and one desired count (associated with the pulse train of unknown frequency) and one standard count (associated with the pulse train of known frequency) in this way is obtained. A measure of the desired frequency can be obtained multiplying the known standard frequency by the ratio between the desired count and the standard count obtained in the two digital counters [1].

The numerical values for the unknown frequency obtained from the process previously described can change hardly in time with each new count in the digital counters, but a global convergence of frequency relative error to zero can be observed for a sufficiently long time. This general and characteristic behavior of results is dependent of ratio between the frequencies of both pulse trains (or similarly the ratio of their periods) and their pulse widths. In computational experiments which is controlling the pulse width in each pulse train, a nonmonotone decreasing characteristic and an alternating convergence to a local minimum of frequency uncertainty is evident, in short measurement time (less than $1 \mathrm{~s}$ for given frequencies) [1].

Assuming: (a) existence of "greatest common measure" between periods of both pulse trains, (b) the frequency measurement by pulse coincidence is formulated like a problem of Diophantine approximation, and (c) decimal notation. The numeric condition that we propose to stop the measurement process is: the desired count (resulting of count the whole periods in the train of the unknown fre- quency) is represented like $10^{r}$, or a one follow by $r$ zeros [1].

A perfect timing signal has a fixed period and duty cycle that never varies over time and a starting point that is fixed in time. However, real word signal exhibit small variations in phase position, period and duty cycle, generally called jitter.

In the method of frequency measurement these variations of the significant instants affect both pulses trains of known and unknown frequency, and the coincidence pulse train.

In the present paper the main goal is to research what natural sources of the phase-noise can affect the measurement process, which way it use to be, and how much it can affect the process of the best coincidence registration.

\section{Jitter general components classification}

A jitter phenomenon is a random variation of the sampling instant. As illustrated in Fig. 2a [3], a random variation of the sampling instant implies a random amplitude variation on the sampled signal (amplitude noise). The amplitude variation is a function of the jitter and the signal derivative, when the input signal is a sine wave sampled at $T_{S}[3]$ :

$x_{o}\left(n T_{S}+\delta_{n}\right)=A \sin \left(2 \pi f_{o}\left(n T_{S}+\delta_{n}\right)\right) \approx x_{o}\left(t_{n}\right)+\left.\frac{\partial x_{o}}{\partial t}\right|_{t_{n}} \times \partial_{n}$

Meanwhile, the amplitude variation is less than timing jitter, as follows from Fig. 2a, and we get an assumption that most important factor able to affect our principle in [1] is a noise in a time domain, or just timing jitter. So, in our furthering simulation of the frequency measurement we will consider this special phenomenon with all related physical characters.

Timing jitter [12] (henceforth referred to as jitter) is defined as short-term non-cumulative variations of the significant instants of a signal from their ideal positions in time [7]. Firstly, the most important aspect in this definition is the non-cumulative character of the jitter. In other words, each pulse of the train has independent from other pulses its phase displacement with arbitrary behavior limited by corresponding distribution law. This fact we take as 


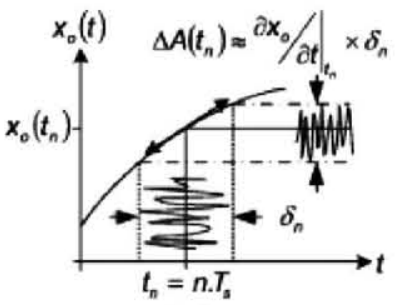

(a)

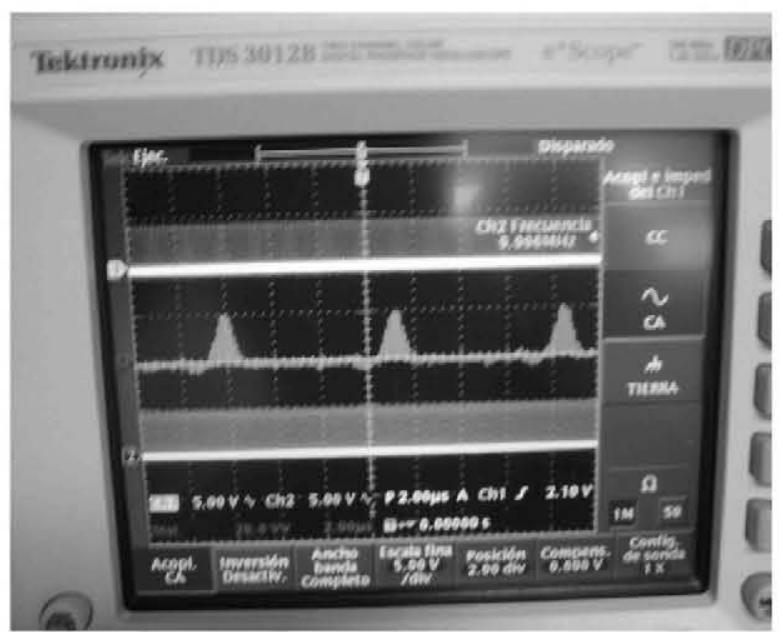

(b)

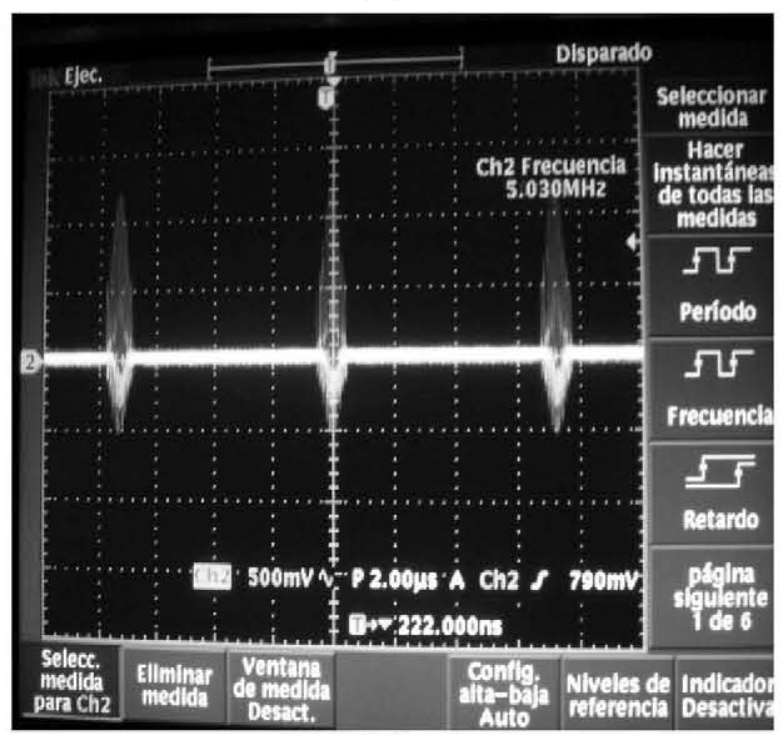

(c)

Fig. 2. Relation between the jitter noise and amplitude noise (a) [3], experimental screenshots for a series of best coincidences in a presence of jitter (b and $c$ ) [1].

one of the most basic in our theoretical model given below, in Section 5.

The presence of jitter in real pulse trains cause the signal vagueness and illegibility in Fig. $2 \mathrm{~b}$ and $\mathrm{c}$. These signal screenshots exactly represent the coincidences trains for- mation during experimentation with two independent pulse trains.

The images presented in Fig. $2 \mathrm{~b}$ and $\mathrm{c}$ are experimentally obtained screenshots of coincidences of two independent pulse trains of unknown and reference frequency detected by prototype circuit presented on Figs. 9 and 10 . These screenshots are shown that the coincidence packages have a non-uniform and noisy shape due to jitter presence.

It is clear evident from both (b and c) screenshots the impossibility to detect by electronic devices both: maximal amplitude of the coincidences train and its true phase position.

It gives only a possibility to find out the true phase position using a special formalism of number theory [11] introduced in [1].

The behavior of pulse phase displacement it is defined by its proper distribution law caused by different sources. It is expedient at this time to consider deeper the possible reasons for jitter generation.

For modeling, it is worldwide recognized [7] that Total Jitter (TJ) consists of two components: Deterministic Jitter (DJ) and Random Jitter (RJ). In time domain, TJ is the sum of the RJ and DJ components [8]. RJ is characterized by a Gaussian distribution. It has been shown that it is theoretically unbounded in amplitude.

DJ consists of several components caused by different and mostly physically-based phenomena, such electronic interference, cross-talk and bandwidth limitation. All DJ subcomponents have a bounded peak-to-peak value that does not increase when more measurement samples are taken [8].

Deterministic jitter has four components: duty cycle distortion (DCD), intersymbol interference (ISI), periodic jitter (PJ) and bounded uncorrelated jitter (BUJ).

DCD and ISI are referred as data correlated jitter, while $\mathrm{PJ}$ and BUJ are referred as data uncorrelated jitter. RJ is unbounded and uncorrelated [8]. In Fig. 2 the block diagram of jitter classification is shows.

\subsection{Random Jitter (RI)}

Random Jitter RJ is caused the common influence of a large number of very small independent contributor or various device-originated noise sources (such as thermal and flicker noise). By the central limit theorem, the distribution of a large number of uncorrelated noise sources approaches a probability Gaussian distribution and is given by $[7,8]$

$J_{\mathrm{RJ}}(x)=\frac{1}{\sigma \sqrt{2 \pi}} e^{-\left(\frac{x^{2}}{2 \sigma^{2}}\right)}$

where $\sigma$ is the standard deviation of the jitter distribution or the RMS value, and $J_{\mathrm{RI}}$ is the probability that leading edge (or trailing edge) will occur at time $x$, where $x$ is the deviation from the mean value of the time reference point (time point related to $50 \%$ amplitude point on pulse edge). In Fig. 3a, is shows the histogram for random jitter. 


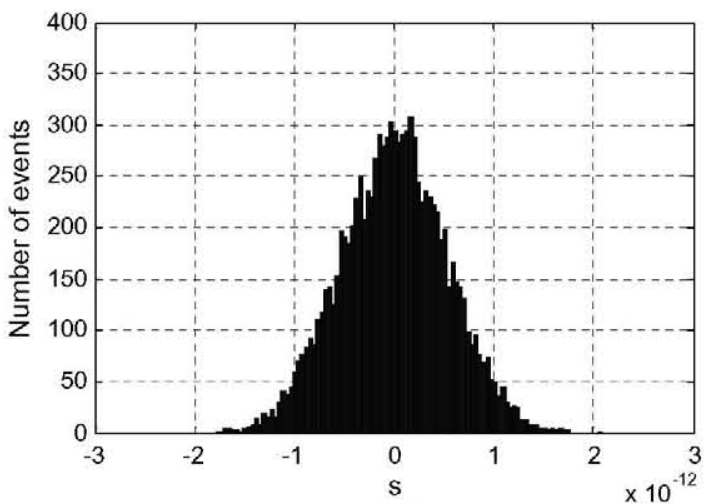

(a)

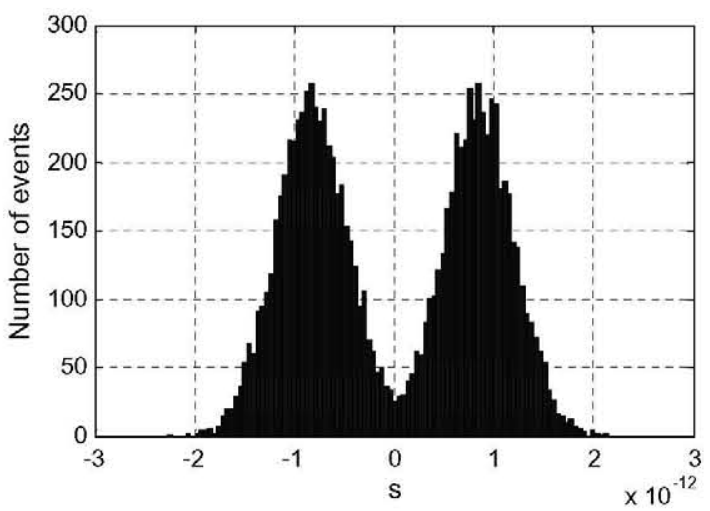

(c)

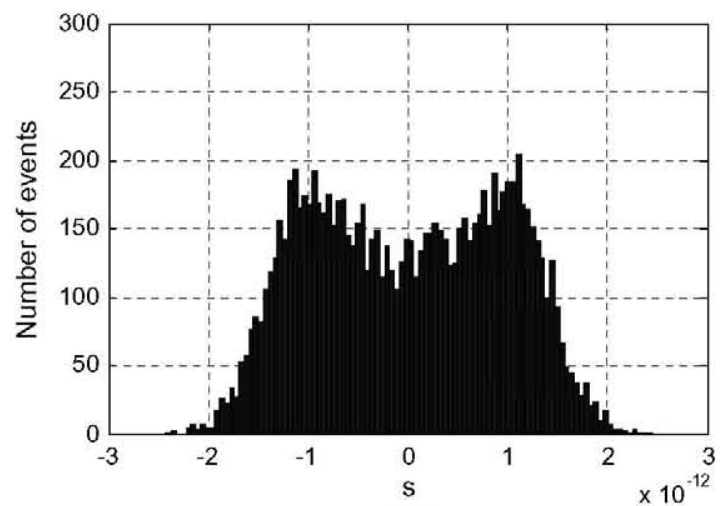

(b)

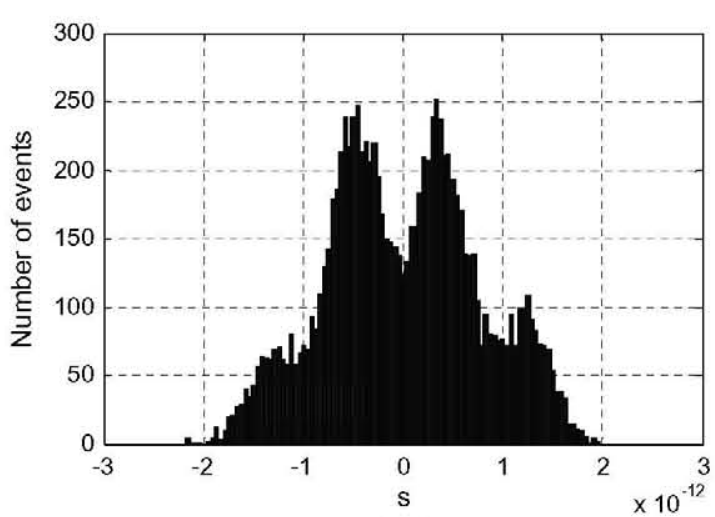

(d)

Fig. 3. Histogram for (a) Random jitter, (b) Sinusoidal periodic jitter with added random jitter, (c) duty cycle distortion with added random jitter y (d) data dependent jitter with added random jitter.

\subsection{Periodic jitter (PJ)}

Periodic jitter denotes periodical timing deviation from the ideal position of a signal that repeat in time, is typically uncorrelated to the data rate or the clock frequency [7]. Electromagnetic interference and crosstalk from some clock line can cause periodic jitter.

The mathematic model of PJ consists of a sum of cosine functions with phase deviation, modulation frequency, and peak amplitude. The model is given by

$\mathrm{PJ}_{T}=\sum_{i=0}^{n} a_{i} \cos \left(\omega_{i} t+\theta_{i}\right)$

where $\mathrm{PJ}_{T}$ denotes the total periodic jitter, $n$ is the number of cosine components, $a_{i}$ is the amplitude in units of time in each tone, $\omega_{i}$ is the angular frequency of the corresponding modulation, $t$ is the time, and $\theta_{i}$ is the corresponding phase [8].

Sinusoidal jitter in time domain produces a probability distribution function given by (defining time zero as the center of the distribution)

$J_{\mathrm{P}_{i}}(x)=\left\{\begin{array}{cc}\frac{1}{\pi \sqrt{a_{i}^{2}-x^{2}}} & |x| \leqslant a_{i} \\ 0 & \text { otherwise }\end{array}\right.$ where $2 a$ is the peak-to-peak width of periodic jitter [5]. In Fig. $3 \mathrm{~b}$ is shows the histogram for sinusoidal periodic jitter with added random jitter.

\subsection{Duty cycle distortion (DCD)}

Duty cycle distortion is often also called pulse width distortion [7], is deviation in the duty cycle value from the ideal value, this equates to a deviation in bit time between a 1 bit (logic 1$)$ and a 0 bit (logic 0$)$. DCD can have several sources. The most common are threshold level offsets and differences in the rising and falling edge characteristics [7].

DCD yields a binomial distribution consisting of two sharp peaks of equal height, unless one separates rising and falling transitions in the measurement. Theoretically those peaks are Dirac delta functions, but in practice random jitter and limited measurement resolution always produce peaks of finite height and finite width. The analytic equation for $D C D$ distribution is the sum of delta functions [7]:

$J_{\mathrm{DCD}}(x)=\frac{\delta(x-a)}{2}+\frac{\delta(x+a)}{2}$ 
where $2 a$ is the peak-to-peak width of the DCD. In Fig. $3 c$ is shows the histogram for duty cycle distortion with added random jitter.

\subsection{Data Dependent Jitter (DDJ)}

Data dependent jitter describes timing errors that depend on the preceding sequence of data bits [7]. DDJ is a predominant form of DJ caused by bandwidth limitations of the system or electromagnetic reflections of the signal $[9,10]$. Since there is always only a limited number of different possible patterns in a data stream of limited length, data dependent timing errors always produce a discrete timing jitter, theoretically DDJ distribution is the sum of two o more delta functions [7]:

$J_{\mathrm{DDJ}}(x)=\sum_{i=1}^{N}\left\{p_{i} \delta\left(x-t_{i}\right)\right\}$

where $\sum_{i=1}^{N} p_{i}=1 \mathrm{~N}$ is number of distinct patterns, $p_{i}$ is the probability of the particular pattern occurring, and $t_{i}$ is the timing displacement of the edge following this pattern. In Fig. 3d is shows the histogram for data dependent jitter with added random jitter.

Each histogram of Fig. 3a-d was independently constructed with data obtained from the jitter simulation in time domain. These simulations are made basing on the known jitters models from the literature sources $[7,8]$ and sections of computation programs described below in Section 6. These four kinds of jitter were simulated in Matlab, using mathematic models introduced in subsections above.

These histograms are obtained like a print for 10,000 points by Matlab native function "hist", or 10,000 events of jitter discrete values according to previous formulas.

Later these forms of jitter were applied in the time domain to the simulated signals as shown on Fig. 7 .

\section{Jitter influence in the frequency measurement method}

Let us consider two trains of narrow pulses $S_{X}(t)$ and $S_{0}(t)$ with period $T_{X}$ and $T_{0}$ respectively, which have pulse width $\tau$. Both pulse trains are generated by detection of zero crossings of two sinusoidal signal of frequencies: $f_{0}$ (standard frequency) and $f_{X}$ (unknown frequency). Suppose that both pulse trains start in phase, i.e. a time shift is 0 . If both pulse trains are applied to the input ports of an ANDgate, an irregular pulse train is formed by pulses from partial and total coincidences is generated, it is shown in Fig. 1.

For frequency measurement, the time intervals $n_{0} T_{0}$ and $n_{X} T_{X}$ are compared (Fig. 1), where $n_{0}$ is the amount of periods $T_{0}$ in the measurement time and $n_{X}$ is the amount of periods $T_{X}$ in the same time interval. Measurement time can be defined by the time interval between the first one pulse of coincidence (start event) after beginning the measurement process, and by any other following pulse of coincidence (stop event).

As it were mentioned in the previous section, $n_{0}$ and $n_{X}$ are the counts of pulses obtained in two independent digital counters.
Mathematical condition to pulse coincidences is

$\left|n_{X} T_{X}-n_{0} T_{0}\right| \leqslant \varepsilon$

where $\varepsilon$ is the acceptable tolerance (reasonable error value between time intervals $n_{0} T_{0}$ and $n_{X} T_{X}$ ) [1,7]. From Eq. (6), measure frequency is expressed by

$\left|f_{X}-\frac{n_{X}}{n_{0}} f_{0}\right| \leqslant \frac{\varepsilon f_{X}}{n_{0} T_{0}}$

And relative error of measurement (frequency offset) $\beta$ can been expressed by

$\beta=\frac{\left|f_{X}-\frac{n_{X}}{n_{0}} f_{0}\right|}{f_{X}} \leqslant \frac{\varepsilon}{n_{0} T_{0}}$.

We can see in (8) that relative error of measurement is limited by the ratio between the acceptable tolerance of the comparison error between time intervals $n_{0} T_{0}$ and $n_{X} T_{X}$ and, the time interval $n_{0} T_{0}$. Value of $n_{0} T_{0}$ is approximately the measurement time (see Fig. 1).

\section{Pulse trains jitter simulation (computational experiment)}

In computational experiments the time reference point of each pulse in both trains of narrow pulses $S_{X}(t)$ and $S_{0}(t)$ are calculated using the equations

$t_{X}\left(m_{X}\right)=\left(m_{X}-1\right) T_{X}+t_{\varphi X}$

and

$t_{0}\left(m_{0}\right)=\left(m_{0}-1\right) T_{0}+t_{\varphi 0}$

for the leading edge. Where $T_{X}$ is hypothetical value of the period of the pulse train with unknown frequency, $T_{0}$ period of the pulse train with known frequency, $t_{\varphi x}$ and $t_{\varphi 0}$ are time intervals associated to the initial phase of each pulse train and, $m_{X}$ and $m_{0}$ are integers numbers, represents the number of pulse in each train in the simulation time window. Graphical interpretation of this parameters is presented in Fig 4a.

For each pulse in both pulse trains, timing variation in time reference point of leading edge are calculated and added to time instants obtained with (9) and (10), depending of the appropriated jitter type, using

$t_{X j}\left(m_{X}\right)=t_{X}\left(m_{X}\right)+\Delta t_{X}$

and

$t_{0 j}\left(m_{0}\right)=t_{0}\left(m_{0}\right)+\Delta t_{0}$

where $t_{X j}\left(m_{X}\right)$ and $t_{0 j}\left(m_{0}\right)$ are the time reference points in each pulse trains affected by jitter, $t_{X}\left(m_{X}\right)$ and $t_{0}\left(m_{0}\right)$ are the time reference points without jitter and, $\Delta t_{X}$ and $\Delta t_{0}$ are timing variation from each other (both are able to get a positive or negative value), assuming as short-term non-cumulative variations.

Coincidences between pulses in the trains are obtained using the coincidence conditions

$\tau_{X}<t_{X j}\left(m_{X}\right)-t_{0 j}\left(m_{0}\right)<\tau_{0}$

where $\tau_{X}$ and $\tau_{0}$ are pulse widths in each train of narrow pulses. 


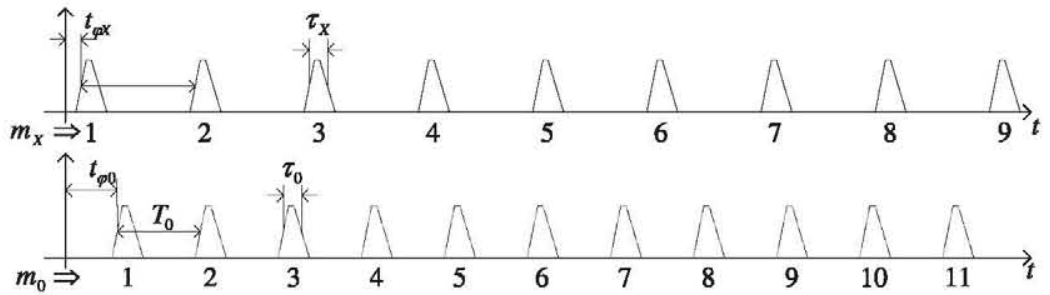

(a)

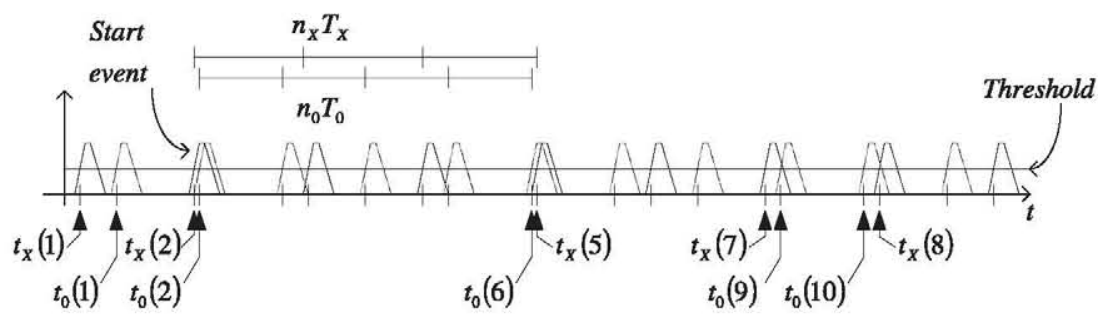

(b)

Fig. 4. Graphic interpretation of parameters used in simulation of pulse coincidence process.

A pulse coincidence is selected as "start event of measurement" in the computational experiments. Using to this coincidence as a counting reference, the number of pulses to the next coincidences is continually counted and, $n_{0}$ and $n_{X}$ is calculated for each next coincidence.

For each next coincidence from the selected coincidence as "start event of measurement" a measure of frequency is calculated by

$f_{X m}=\frac{n_{X}}{n_{0}} f_{0}$

and the relative error of measurement using (8).

\section{Experimental results}

In [1] it is mentioned that the best approximation ratio $\frac{n_{X}}{n_{0}}$ appears several times during relatively short time of $f_{X}$ and $f_{0}$ pulse trains observation. It is extremely clear shown in Fig. 7 of [1, p. 142]. Es evident from this figure (see Fig. 5), for used in simulation values of $f_{X}$ and $f_{0}$ the best approximation (with theoretical error equal to zero) appears 5 times during only $1 \mathrm{~s}$. It is essential note that this quantity will only increase proportionally with both frequencies enhancement, so for the higher frequencies absolute values the best approximation can be carried out faster. It means that with frequencies enhancement the measurement time becomes less. It is extremely important for multiples applications of sensors which are sensitive to physical parameters converted into fast changes of frequency.

The first one of these best approximations registered in only $0.17 \mathrm{~s}$. This value appears in Fig. 6 which is a zoom of Fig. 5 on time interval $0.1-0.2 \mathrm{~s}$. In [1] it is strictly shown, that this value is equal to the desired frequency value, and the uncertainty of this measurement depends only on reference frequency source own noise.

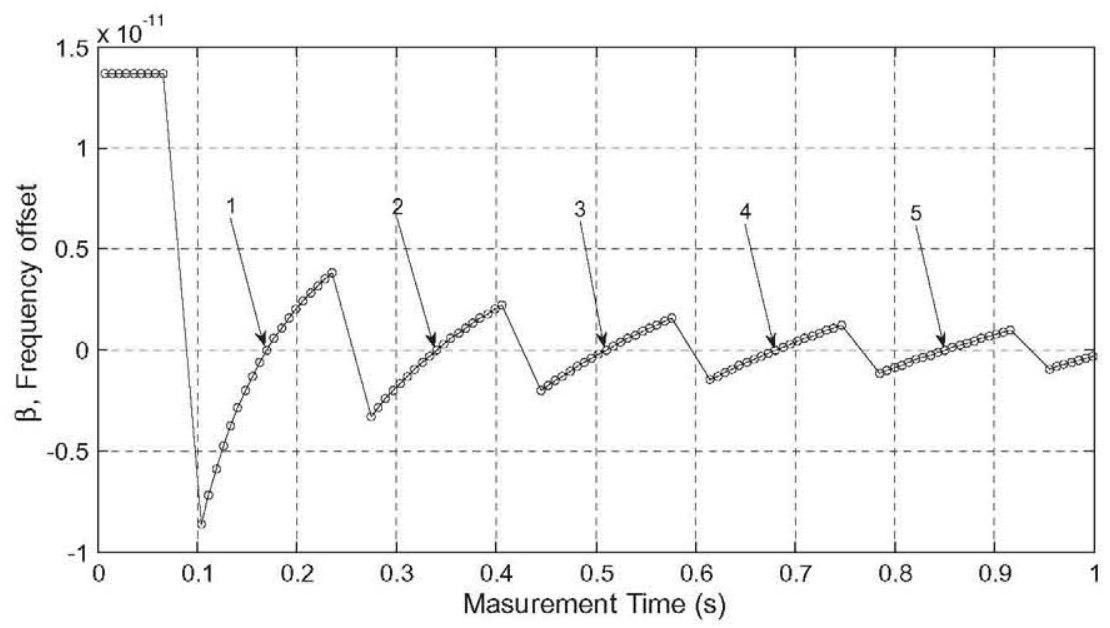

Fig. 5. Frequency count results for a series of best coincidences (citation of Fig. 7 in [1]). 


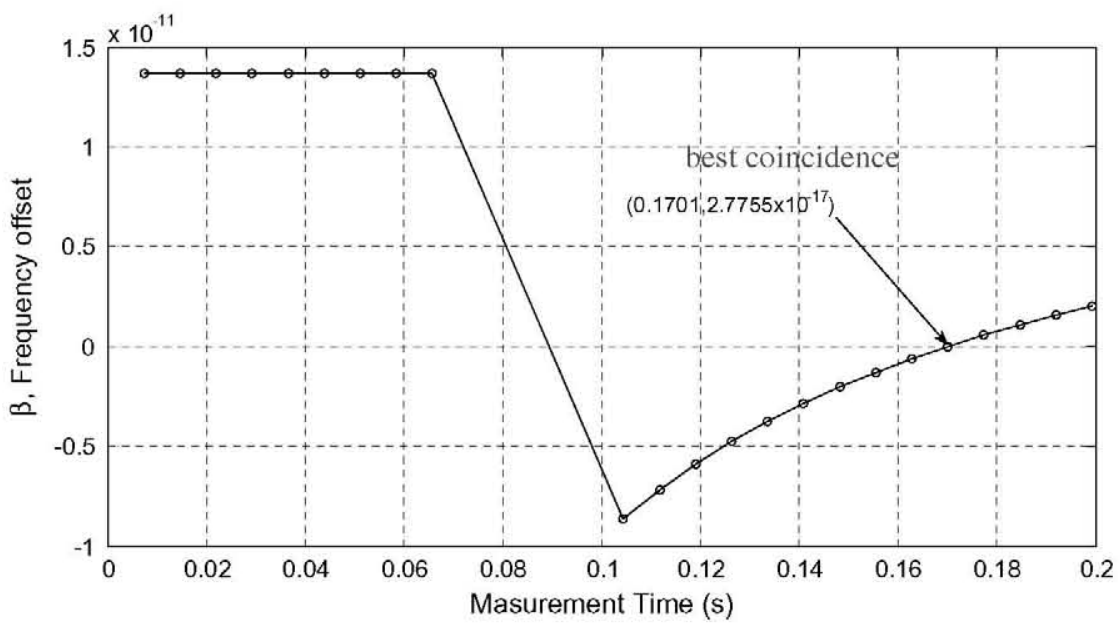

Fig. 6. Frequency offset for the best coincidences selection (the mediant's numeric value).

The best coincidences, obtained under condition (6) with $\varepsilon=1 \times 10^{-12}$ are presented in Fig. 5. In this graphic, we can observe a convergence by the left and a divergence by the right around $0.17 \mathrm{~s}$. This condition is repeated with time, and in Fig. 5 we can see such a situation five times and five points where frequency offset $\left|\beta_{x}\right|$ is minimum, for a simulation time of $1 \mathrm{~s}$.

Our simulation is considering the flow of discrete events like coincidence along time axis the discrete pulses of unknown and standard trains. So, the real events of simulation in these graphics are points, not lines. And when we tell "the minimum is really for $0.17 \mathrm{~s}$ " it means that in simulation point closest to $0.17 \mathrm{~s}$ for example in Fig. 6 (Fig. 6 is a zoom of this point in Fig. 5) theoretical error of measurement is strictly equal to zero. Than we can conclude that between various good approximations to true value are some of them, which are the best ones on the interval under consideration. The lines in Figs. 5 and 6 we need to underline the alternate (but not chaotic) character of coincidence process.

However, in [1] the proof of this result was deduced in jitter absence. It is strongly need at this moment by process simulation to check the possible influences of arbitrary phase variations in Figs. 5 and 6 behavior.

The computational experiments in the present paper have the goal to review which way the jitter presence in real pulse trains can affect the best approximation criterion.

For this purpose in Matlab firstly were generated in time domain all kinds of jitter from different sources with possibility to link them to two considered pulse trains. It was all kinds of jitter from Fig. 3. The results of jitter simulation in real time under formulas of Section 5 are given in Fig. 7.

The first row of Fig. 7 represents the simulation in time domain of the random jitter, shown in Fig. 3a; the second row of Fig. 7 represents sinusoidal periodic jitter with added random jitter in Fig. 3b, the third row of Fig. 7 represents duty cycle distortion with added random jitter in Fig. $3 \mathrm{c}$ and the fourth row is data dependent jitter with added random jitter from Fig. $3 \mathrm{~d}$.
In the simulation program was provided option to apply all these kinds of jitter distributions law to both of pulse trains, - of the unknown and the standard frequencies-, independently (see the example program fragments):

\begin{tabular}{|c|c|}
\hline $\begin{array}{l}\text { randn('state', } \\
\quad \text { sum }(100 * \text { clock }))\end{array}$ & $\begin{array}{l}\text { \% Initialize randn to a } \\
\text { different state each time } \\
\text { (normal distribution). }\end{array}$ \\
\hline$n=1$ & $\begin{array}{l}\text { \% Index for number of } \\
\text { coincidence. }\end{array}$ \\
\hline$m o=1$ & $\begin{array}{l}\% \text { Index for number of } \\
\text { pulse in reference train. }\end{array}$ \\
\hline$m x=1$ & $\begin{array}{l}\text { \% Index for number of } \\
\text { pulse in unknown train. }\end{array}$ \\
\hline \multicolumn{2}{|l|}{$\operatorname{coin}=[]$; } \\
\hline to $=(m o-1) * T o+d t o$ & $\begin{array}{l}\text { \% Significant instants for } \\
\text { rising edge in reference } \\
\text { train (Eq. (9)). }\end{array}$ \\
\hline$t x=(m x-1)^{*} T x+d t x$ & $\begin{array}{l}\% \text { Significant instants for } \\
\text { rising edge in unknown } \\
\text { train. (Eq. (10)). }\end{array}$ \\
\hline $\begin{array}{l}{[\text { Tjio, Tjso] }=\operatorname{calcji1}(A o, \text { Apo, }} \\
\text { fno, k1o, Bo, k2o, Po, Do, } \\
\text { k3o, tijio); }\end{array}$ & $\%$ Jitter calculation for \\
\hline $\begin{array}{l}{[T j i x, \text { Tjsx] }=\text { calcji1 }(A o, A p x} \\
\quad \text { fnx, } k 1 x, B x, k 2 x, P x, D x \\
k 3 x, \text { tijix); }\end{array}$ & $\%$ Both trains. \\
\hline to $=$ to + Tjso & $\begin{array}{l}\% \text { Add jitter to significant } \\
\text { instant in reference train. }\end{array}$ \\
\hline$t x=t x+T j s x$ & $\begin{array}{l}\% \text { Add jitter to significant } \\
\text { instant in unknown train. }\end{array}$ \\
\hline $\begin{array}{l}\text { while to } \leqslant T \operatorname{sim} \\
\text { if to } \geqslant t x\end{array}$ & \\
\hline $\begin{array}{l}\text { if } 1 e-16<\operatorname{tau} x- \\
(\text { to }-t x) \\
\quad \operatorname{coin}(n,:)=[m o m x] \\
n=n+1\end{array}$ & $\begin{array}{l}\text { \% First part of coincidence } \\
\text { condition (Eq. (13)) }\end{array}$ \\
\hline
\end{tabular}

(continued on next page) 


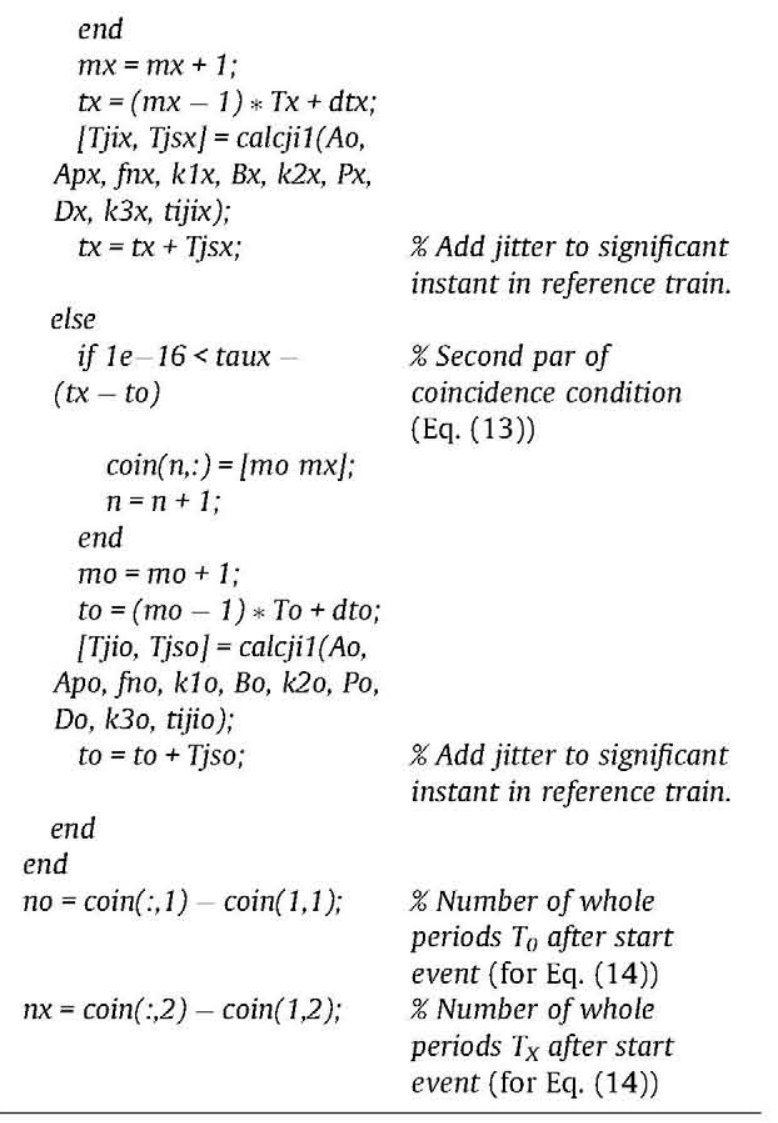

To simulate random jitter, we will use the native Matlab function randn, initialize to a different state each time of simulation, with a RMS value of 0.7 ps. ([8], p.140).

To simulate periodical jitter plus random jitter, is used a single-tone sinusoidal with peak-to-peak value of $10 \mathrm{ps}$ and frequency of $5 \mathrm{MHz}$ ([8], p.140).

$$
\begin{aligned}
& T j i=A p * \sin (2 * p i * f n * \text { rand }) ; \\
& T j s=T j i+\operatorname{randn}(1) * A o / k 1 ;
\end{aligned}
$$

An additional pseudo uniform distributed variable is added in the model (rand: Matlab native function) to emulate the position and mobility of the source of interference, because this interference plays an important role in formation of jitter and the behavior of its source is random.

To simulate duty cycle distortion plus random jitter, first a two impulse function is generate using a pseudo uniform distributed variable (Matlab native function) an the code

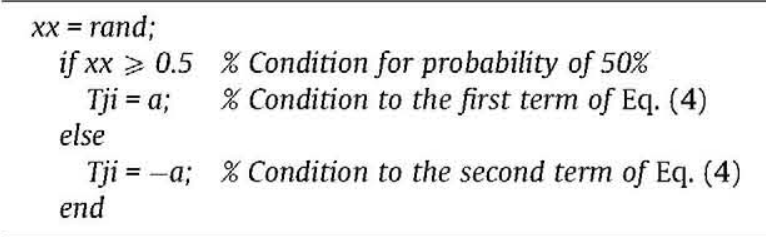

Then, due the Matlab native function rand returns values drawn for the standard uniform distribution on the open interval $(0,1)$, Tji only take values $a$ or $-a$ with approximated probability of $50 \%$ each other. After, a pseudo normal distributed variable is added with RMS value like a fraction of the RMS value of random jitter.

$T j s=T j i+\operatorname{randn}(1) * A o / k 2$;

A similar algorithm is used for simulation data dependent jitter. In this case the open interval $(0,1)$ is $N$ different patterns so that the length of each subinterval is equal to the probability desired, and each point in the interval is assigned the corresponding value. In this are used only four intervals, but we can five. Finally random jitter is added.

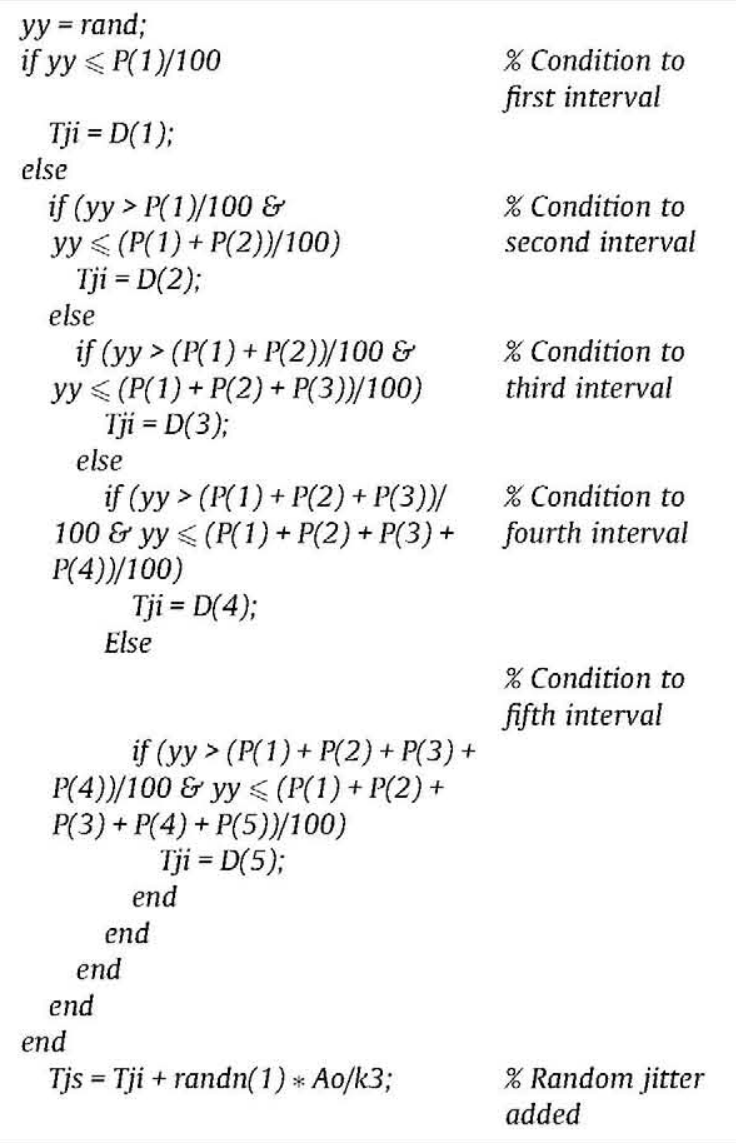

Analysis of Fig. 3d shows that this typical case no have direct application in our experimentation (or, at least, is the less affecting our task): this jitter affects discrete data transferring system, meanwhile in the case under consideration we have likely other phenomena, the analogue drift of the integer measured value. Moreover, according to jitter classification in [8] (see Fig. 1 on the page 135 of [8]) these authors recommends only two components as applicable in our case (PJ and BUJ). However, we still simulating more cases, because in our opinion it is probable find out more sources with influence on our method functioning. 

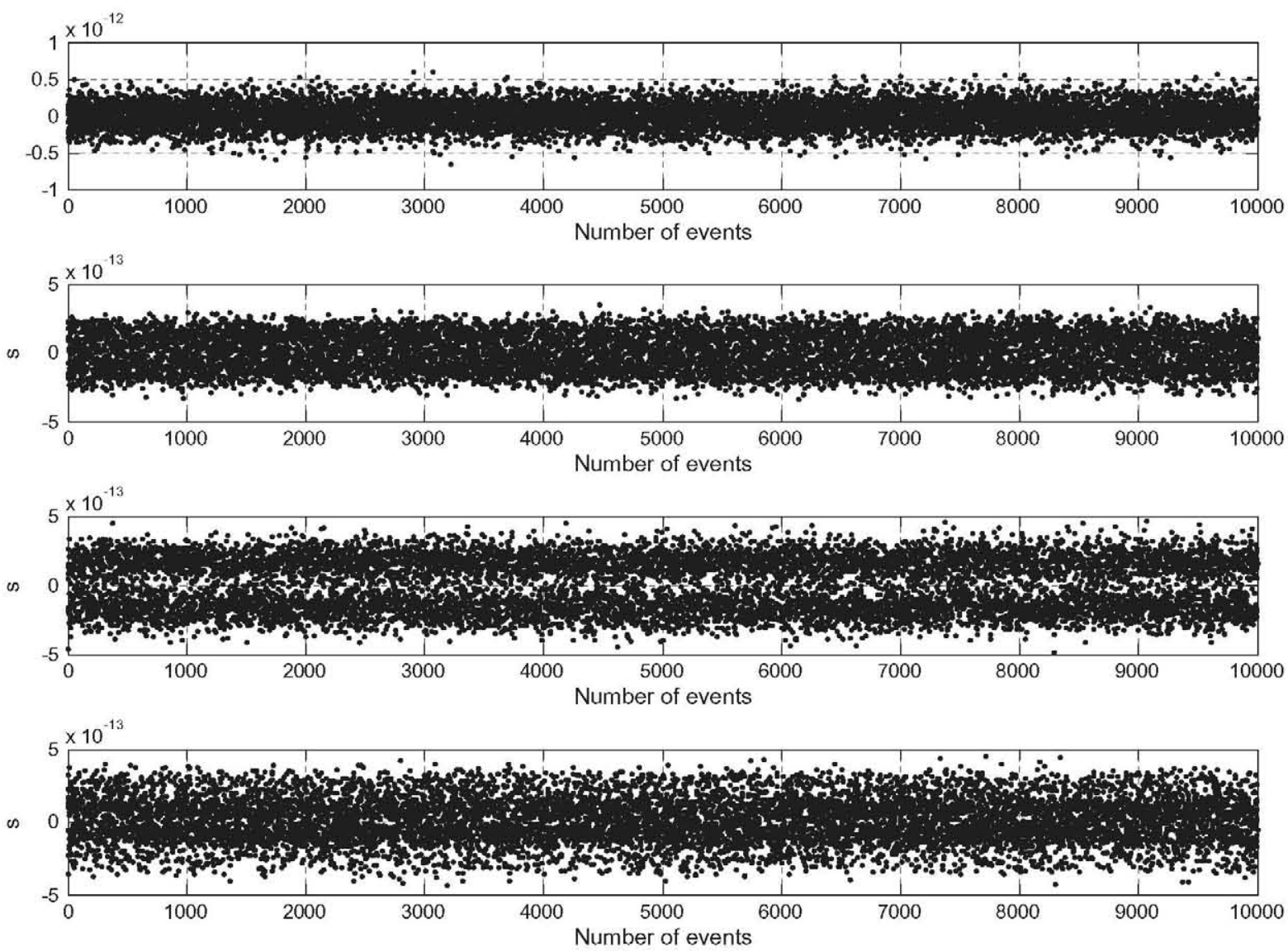

Fig. 7. Generation in time domain the different kinds of jitter from Fig. 3.

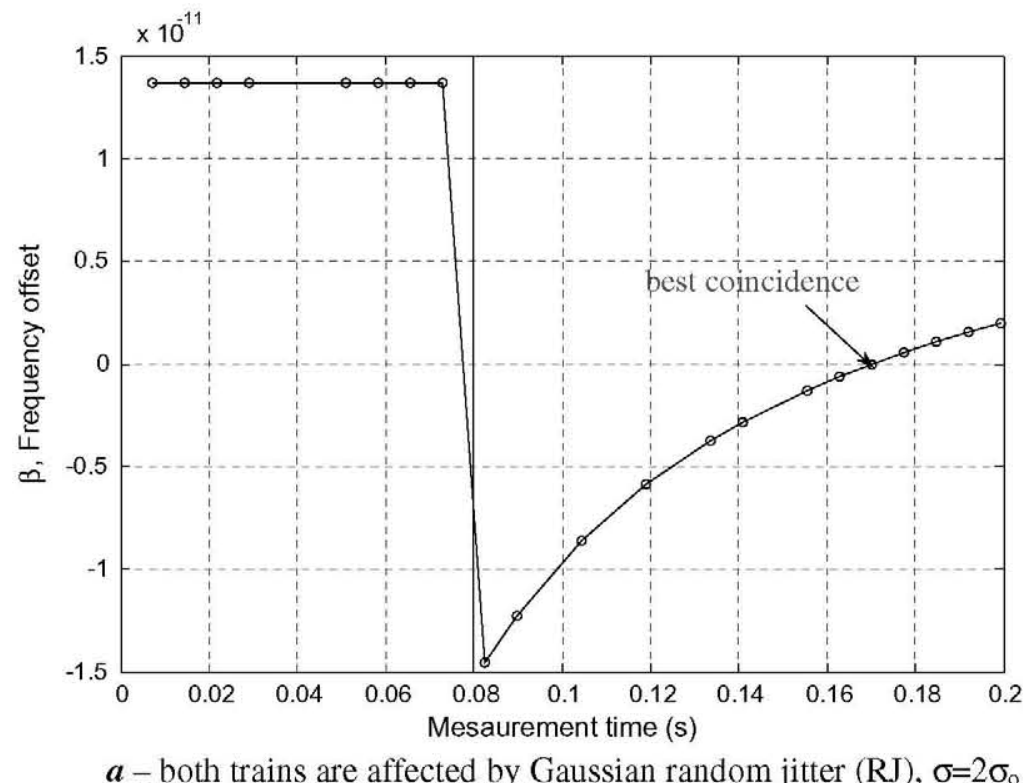

Fig. 8. Simulation results for the different kinds of noise affecting two bearing frequencies. (a) both trains are affected by Gaussian random jitter (RJ), $\sigma=2 \sigma_{0}$. (b) standard affected with RJ and unknown with mixed jitter (PJ + RJ). (c) standard affected with RJ and unknown with mixed jitter (DCD + RJ). (d) standard affected with RJ and unknown with mixed jitter (DDJ + RJ). 


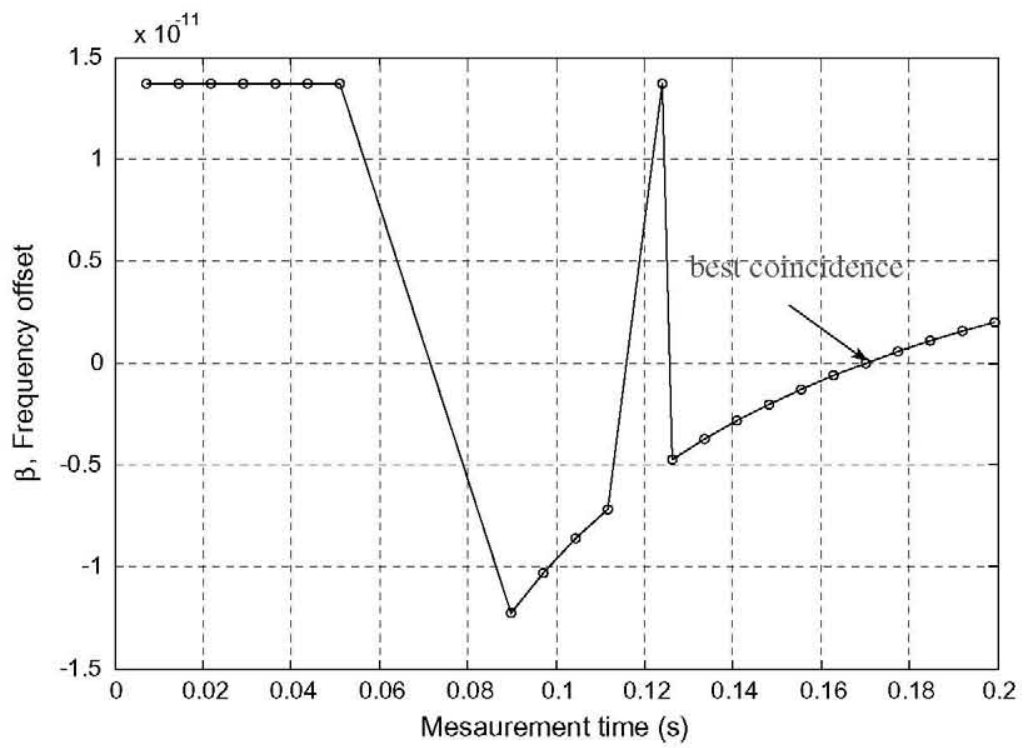

$\boldsymbol{b}$ - standard affected with RJ and unknown with mixed jitter (PJ+ RJ)

Fig. 8 (continued)

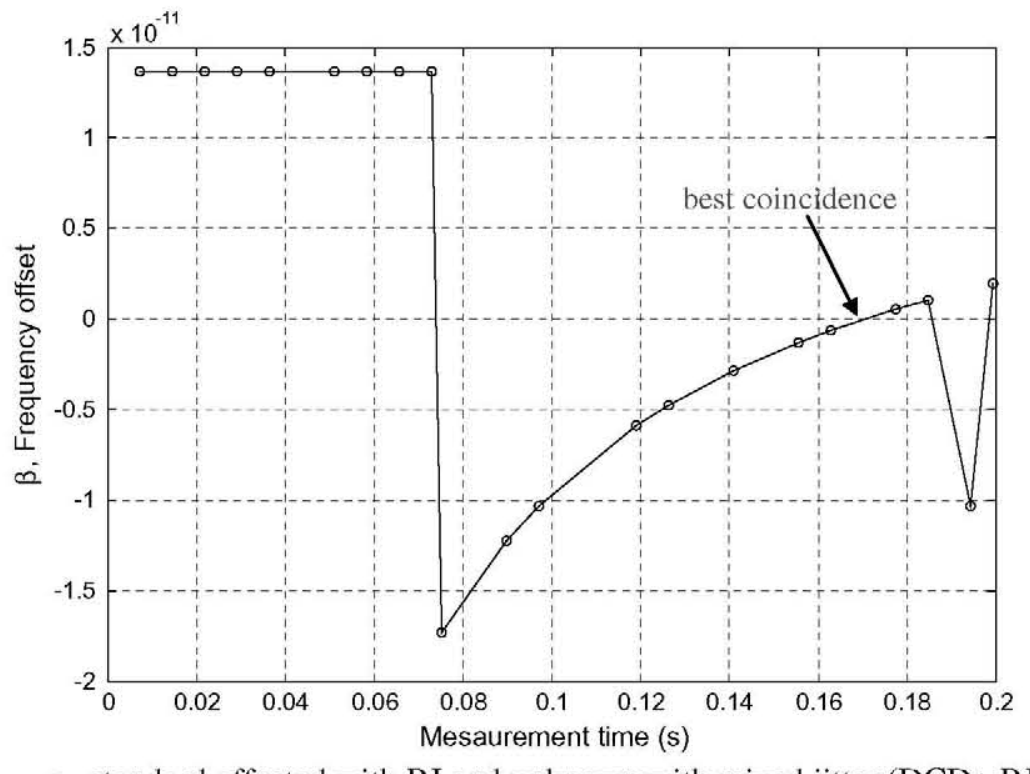

$c$ - standard affected with RJ and unknown with mixed jitter (DCD+ RJ)

Fig. 8 (continued)

So, because of this reason the furthering simulations were applied for all four kinds of jitter, considering as most probable three first cases of Fig. 3.

At the same time, there was maintained an algorithm to detect pulses coincidences, and to count the pulse number where it appears. The results of such simulations for introduced kinds of jitter are presented in Fig. 8a-d:

Let compare the occurred variations in process with ideal un-jittered process, represented in Fig. 6. In all simulated cases (Fig. 8a-d) it is clear shown that jitter influence affects significantly the package of coincidence pulses (group of impulses), however the position of the referred best coincidence always are the same.

Moreover, this value is constant and not depends on the kind of provided revolting factor.

Also is useful to analyze the simulation results due to influence of the different combinations of jitter on the general behavior of Fig. 5. These possible combinations are resulted in Table 1. With green ovals are marked splashes (variations) in normal process behavior caused by jitter. But with red circle always marked the position of the best coincidence derived from condition in [1]. 


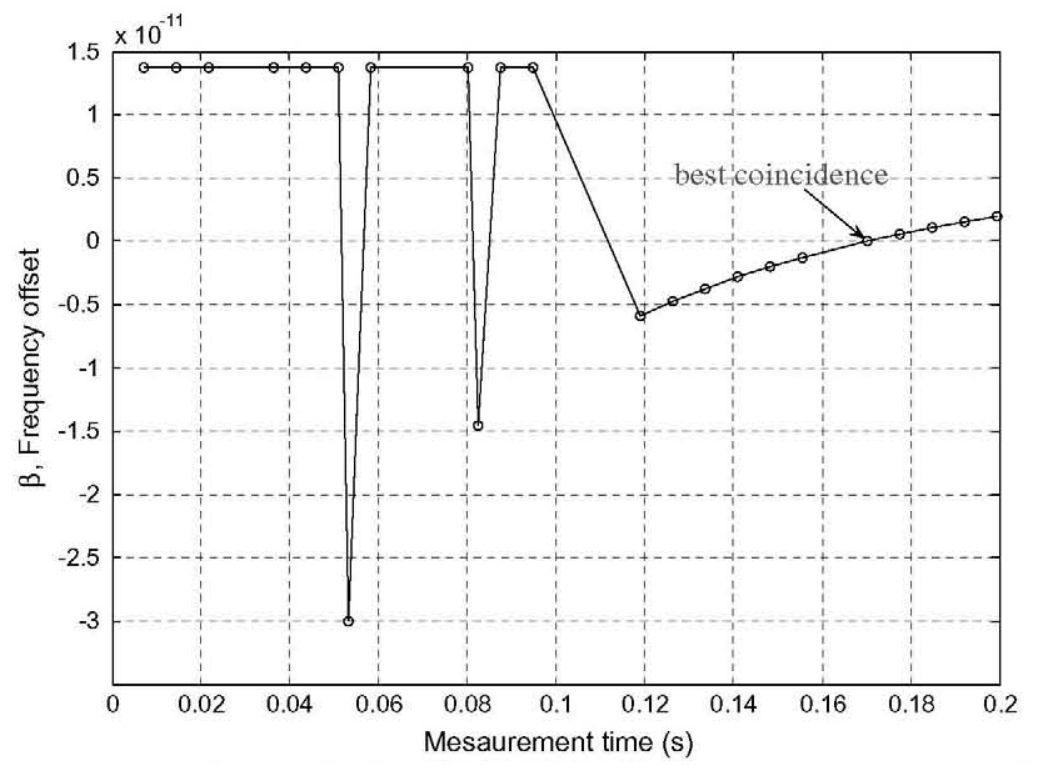

$\boldsymbol{d}$ - standard affected with RJ and unknown with mixed jitter (DDJ + RJ)

Fig. 8 (continued)

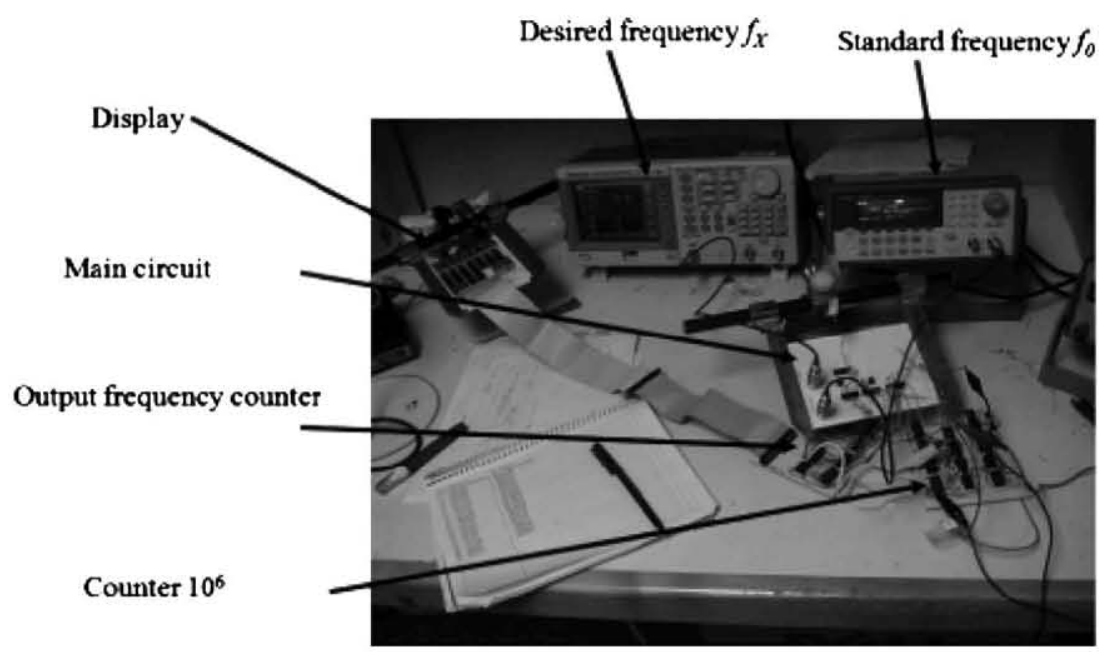

Fig. 9. Frequency meter prototype test circuit.

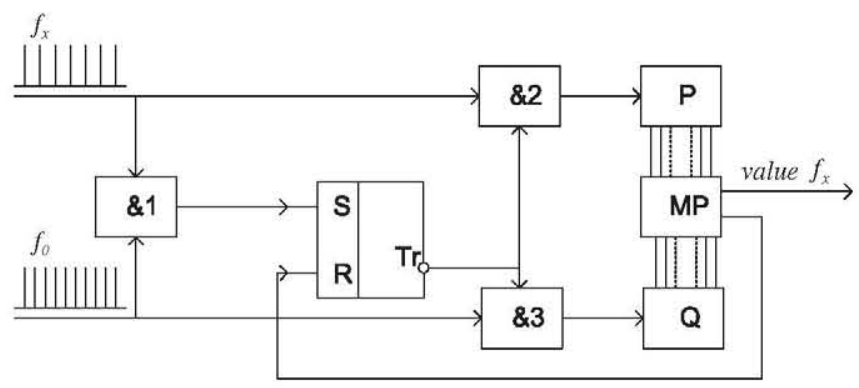

Fig. 10. The frequency meter block diagram for frequency measurement through direct comparison with a reference. Gates $\& 1,2,3$ are schemes of coincidences (\&-gates); Tr is the trigger; P, Q are $T_{x}, T_{0}$ - periods counters of unknown frequency $f_{x}$ and reference frequency $f_{0}$, and the MP is microprocessor. 
Table 1

Different combinations of jitter application to both (reference and unknown) pulse trains in computational model of real process.

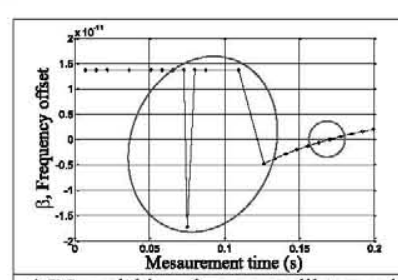

a) RJ model in reference oscillator and model in oscillator under test.

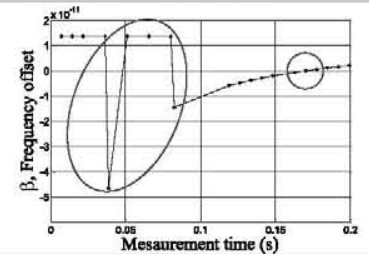

c) RJ model in reference oscillator and DDJ+RJ model in oscillator under test.

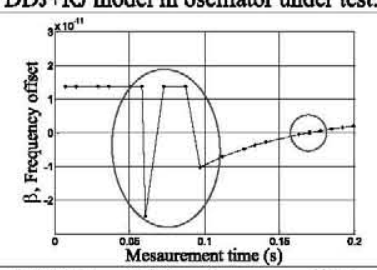

e) $\mathrm{PJ}+\mathrm{RJ}$ model in reference oscillator and RJ model in oscillator under test.

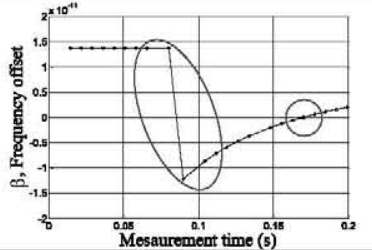

() PJ+RJ model in reference oscillator test.

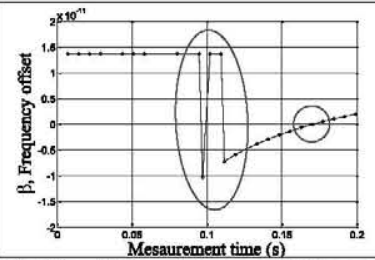
b) RJ model in reference oscillator and
$\mathrm{PJ}+\mathrm{RJ}$ model in oscillator under test.



Mesaurement time (s)

$\mathrm{DCD}+\mathrm{RJ}$ model in oscillator under test.

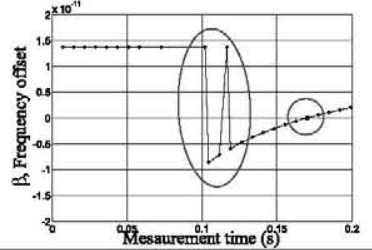

f) PJ+RJ model in reference oscillator and f) PJ+RJ model in reference oscillator
PJ+RJ model in oscillator under test.

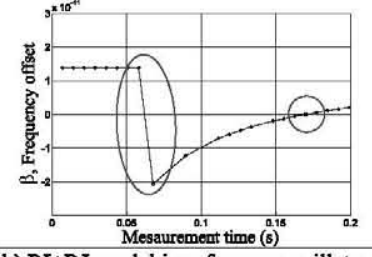

h) PJ+RJ model in reference oscillator an $\mathrm{DCD}+\mathrm{RJ}$ model in oscillator under test.

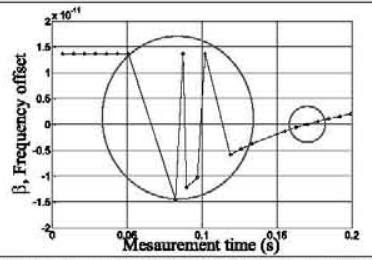

a) DDJ+RJ model in reference oscillat and $\mathrm{RJ}$ model in oscillator under test.

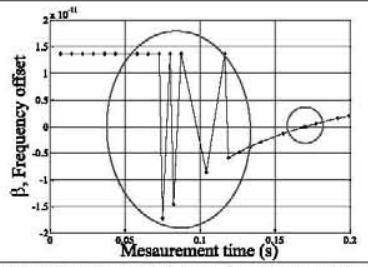

c) DDJ+RJ model in reference oscillator and DDJ+RJ model in oscillator under test.

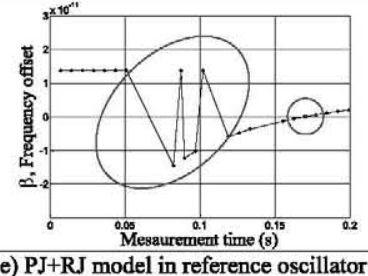

e) PJ+RJ model in reference oscillator an RJ model in oscillator under test.

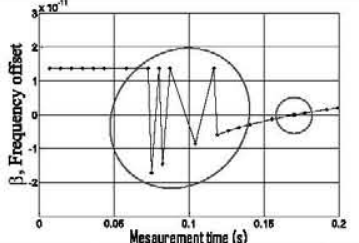

g) DCD+RJ model in reference oscillator and DDJ+RJ model in oscillator under test.

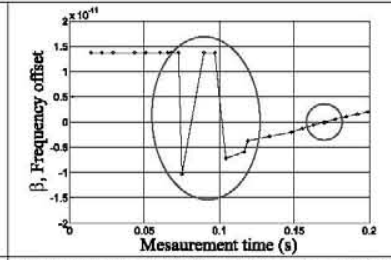

b) DDJ+RJ model in reference oscillator an PJ $+R J$ model in oscillator under test.

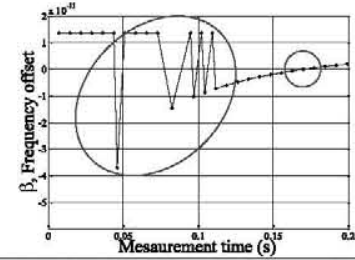

d) DDJ+RJ model in reference oscillator an $\mathrm{DCD}+\mathrm{RJ}$ model in oscillator under test.

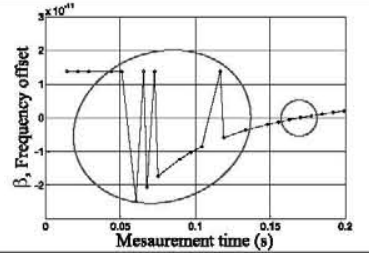

f) PJ+RJ model in reference oscillator an $\mathrm{PJ}+\mathrm{RJ}$ model in oscillator under test.

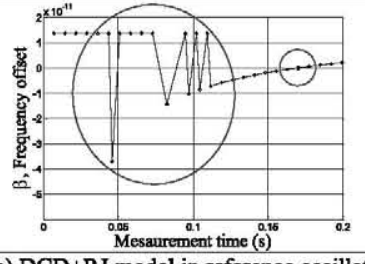

h) DCD+RJ model in reference oscillator

and DCD+RJ model in oscillator under test. 
However, analyzing all the variations in Table 1, these cases also proofs that mentioned splashes and imperfections can affects only pulses packages at its boundaries. Position of the center never is variable, and it is defined exclusively by process nature, number theory laws, and numerical condition [1] at the same time.

\section{Discussions}

As shown by computational experiment results, the best approximation condition, derived in [1] it is invariant (independent) to jitter. This statement it is rigorous for any kind of jitter. This main contribution of the present research gives more backgrounds for the method of the fast frequency measurement implementation, especially for various practical applications [16,17] of the sensors sensitive in frequency domain, or repetitive measurement processes with possibility to apply the principle of continuous coincidences of two independent scales $[18,19]$.

It is only one circumstance maybe critical in the presented above: all these simulation results are derived in assumption stated at the beginning of Chapter 3, that timing jitter has non-cumulative variations. There is no one another suggestion about different jitter behavior in most fundamental references in this field [7,8,12]. However, for completed analysis of all noise effects at frequency measurement according to [1], in our opinion it is desirable the research of possible accumulative thermal drift of pulses in train at the temperature changes. It is clear that such task more proper for real experimentation than for computer simulation. For this goal was made an experimental prototype of the measuring system in Fig. 9.

The simplified functional diagram of a frequency meter of Fig. 9 is presented in Fig. 10 [1, p. 139]. Pulse signals with frequencies $f_{X}$ and $f_{0}$ inputs into three \&-gates. Prior to the first coincidence in the gate \&1 the trigger Tr prevents elements $\& 2, \& 3$ from being active.

After the occurrence of a coincidence, the counters $P$ and $Q$ keep a count of the pulses of both frequencies until counter $\mathrm{P}$ receives a result in the form of $\sum_{m} \mathrm{P}_{n}=1 \times 10^{r}$. This signal results in feedback that resets the trigger to its initial state. The measurement is completed.

We assert that at the end of the measurement, the readout result in the counter $\sum_{m} Q_{n}$ is the best proportional approximation of the measured frequency's true value on the given interval of time.

Influence analysis of thermal long-term accumulative drift of pulses from their ideal positions in time will be a subject for our future publications.

\section{Conclusions}

Assuming the analysis of simulation results of the possible practical jitter influence for frequency measurement method introduced in [1] we can declare the next.

- Random and deterministic components of jitter are modeled, and each one of them affects in a different way the general behavior of pulses on the two pulse trains used in the method, and the train resulted as their intersections in time domain.

- Under inspection in this work were provided in computational simulation four different known and described in recent literature sources of jitter.

- The results of simulation proofs the invariance (independence) of best approximation position on any described in literature sources of short-term non-cumulative variations of the significant instants of a signal from their ideal positions in time domain.

\section{Acknowledgments}

Particular results of this investigation herein described were reported in several congresses [13-15], we are grateful to congress organizers for fruitful discussions. The authors dedicate this article to the grateful memory of our teacher, Dr. Valentin Tyrsa; and would like to thank to anonymous reviewers for valuable comments and remarks.

\section{References}

[1] Daniel Hernández Balbuena, Oleg Sergiyenko, Vera Tyrsa, Larysa Burtseva, Moisés Rivas López, Signal frequency measurement by rational approximations, Measurement, vol. 42, no. 1, Elsevier, 2009, pp. 136-144. doi:10.1016/j.measurement.2008.04.009. ISSN: 02632241.

[2] D.W. Allan, Time and frequency (time-domain) characterization, estimation, and prediction of precision clocks and oscillators, IEFE Trans. Ultrason. Ferroelectr. Freq. Control 34 (752) (1987) 647-654.

[3] G. Monnerie et al., Internal Jitter noise measurement procedure of a switched capacitor circuit, Measurement, vol. 42, no. 1, Elsevier, 2009, pp. 1-8.

[4] V.E. Tyrsa, Error reduction in conversion of quantities to digitalized time intervals, Measurement Techniques, vol. 18, no. 3, Springer, New York, 1975, pp. 357-360.

[5] J.C. Fletcher, Frequency measurement by coincidence detection with standard frequency, US Patent 3, 924,183, 1975

[6] Z. Wei, The greatest common factor frequency and its application in the accurate measurement of periodic signals, in: Proceedings of the IEEE Frequency Control Symposium, 1992, pp. 270-273.

[7] W. Maichen, Digital Timing Measurement, From Scope and Probes to Timing and Jitter, FRET 33, Frontier in Electronic Testing, Springer, Netherland, 2006, p. 240. ISBN: 0-387-31418-0.

[8] K.K. Kim et al., Analysis and simulation of jitter sequences for testing serial data channels, IEEE Trans. Ind. Inf. 4 (2) (2008) 134-143.

[9] J. Buckwalter et al., Predicting data-dependent jitter, IEEE Trans. Circ. Systems-II: Express Briefs 51 (9) (2004) 453-457.

[10] B. Analui et al., Data-dependent jitter in serial communications, IEEE Trans. Microwave Theory Tech. 53 (11) (2005) 3388-3397.

[11] Robert Daniel Carmichael, The Theory of Numbers and Diophantine Analysis, Courier Dover Publications, Dover Phoenix Editions, 2004, p. 118 . ISBN: 0486438031 .

[12] 1.S. Gonorovsky, Radio Circuits and Signals, MIR Publishers, Moscow, 1981, p. 639 (translated to English).

[13] B. Daniel Hernández, L. Moisés Rivas, Larisa Burtseva, Oleg Sergiyenko, Vera Tyrsa, Method for phase shift measurement using farey fractions, in: IEEE-LEOS Proceedings Multiconference on Electronics and Photonics (MEP-2006), Guanajuato, Mexico, Guanajuato, November, 7-10, 2006, pp. 181-184. ISBN: 1-42440627-7, ISSN/Library of Congress Number 2006932321.

[14] Daniel Hernandez Balbuena, Oleg Sergiyenko, Vera Tyrsa, Larisa Burtseva, Frequency measurement method for mechatronic and telecommunication applications, in: IEEE-IES Proceedings International Symposium on Industrial Electronics (ISIE-2008), Cambridge, United Kingdom, June 30-July 2, 2008, pp. 1452-1457. ISBN: $978-1-4244-1665-3$, ISSN/Library of Congress Number 2007936380

[15] Daniel Hernandez Balbuena, Oleg Sergiyenko, Vera Tyrsa, Larisa Burtseva, Method for fast and accurate frequency 
measurement, in: Proceedings of 16th IMEKO $\mathrm{TC}_{4}$ Symposium Exploring New Frontiers of Instrumentation and Methods for Electrical and Electronic Measurements, Florence, Italy, 20-22 September, 2008, pp. 367-373 [CD-ROM]. ISBN: 978-88903149-3-3.

[16] Fabián N. Murrieta Rico, O. Yu Sergiyenko, V.V. Tyrsa, D. HernándezBalbuena, W. Hernandez, Frequency domain automotive sensors: resolution improvement by novel principle of rational approximation, in: Proceedings of IEEE-ICIT International Conference on Industrial Technology (ICIT'10), 14-17 March, Viñadel-Mar, Valparaiso, Chile, 2010, pp. 1293-1298. ISBN: 978-1-42445697-0/10.

[17] Oleg Starostenko, Vicente Alarcon-Aquino, Wilmar Hernandez, Oleg Sergiyenko, Vera Tyrsa, Algorithmic Error Correction of Impedance
Measuring Sensors, vol. 9, no. 12, MDPI, Sensors, 2009, Basel, Switzerland, pp. 10341-10355. doi:10.3390/s91210341. ISSN: 1424-8220.

[18] Moisés Rivas López, Oleg Sergiyenko, Vera Tyrsa, Wilmar Hernandez Perdomo, Daniel Hernández Balbuena, Luis Devia Cruz, Larisa Burtseva, Juan Iván Nieto Hipólito, Optoelectronic method for structural health monitoring, International Journal of Structura Health Monitoring, vol. 9, no. 2, SAGE Publications, March, 2010, pp. 105-120. Issue Online, September, 24, 2009. doi:10.1177/ 1475921709340975. ISSN: 1475-9217.

[19] O. Yu. Sergiyenko, Optoelectronic system for mobile robot navigation, Optoelectronics, Instrumentation and Data Processing, Vol. 46, no. 5, Springer/Allerton Press, Inc., October, 2010, pp. 414 428. doi: $10.3103 /$ S8756699011050037. ISSN: 8756-6990. 